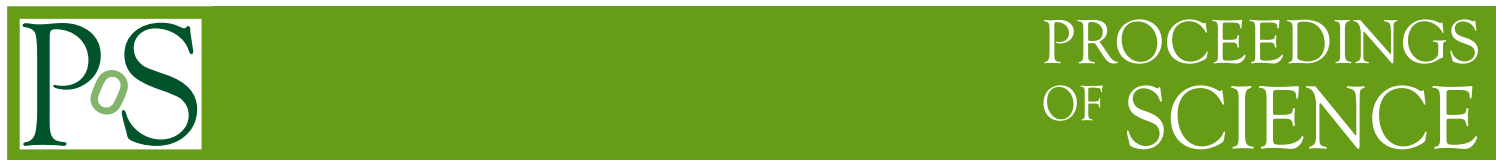

\title{
Theoretical Status of Neutrino Physics
}

\section{Marco Drewes*}

Technische Universität München, James Franck Straße 1, D-85748 Garching, Germany

E-mail: marco.drewes@tum.de

I summarise my viewpoint on the theoretical status of neutrino physics. The key question remains what is the origin of neutrino masses. Their explanation in the framework of renormalisable relativistic quantum field theory necessarily requires the existence of new physical states. These new states may also be responsible for other unexplained phenomena in particle physics and cosmology. After a brief introduction, I focus on scenarios in which the neutrino masses are generated by the type-I seesaw mechanism and review the phenomenological implications of different choices of the seesaw scale.

16th International Workshop on Neutrino Factories and Future Neutrino Beam Facilities - NUFACT2014, 25 -30 August, 2014

University of Glasgow, United Kingdom

\footnotetext{
* Speaker.
} 


\section{Why are neutrinos so interesting?}

The Standard Model of particle physics and theory of general relativity form the basic pillars of modern physics. While there are many emergent phenomena in nature the understanding of which poses a great challenge due to their complexity, only four observations have been confirmed ${ }^{1}$ that cannot in principle be understood this framework [1]: ${ }^{2}$

(1) neutrino flavour oscillations,

(2) the baryon asymmetry of the universe (BAU), i.e. the tiny excess of matter over antimatter in the early universe, which explains the presence of matter in the cosmos at present time as the "leftover" after mutual annihilation of all antimatter with matter, ${ }^{3}$

(3) the composition and origin of the Dark Matter (DM) that appears to make up most of the mass of galaxies and galaxy clusters and

(4) the hot big bang initial conditions, in particular the overall homogeneity and isotropy of the early universe seen in the cosmic microwave background (flatness and horizon problems). ${ }^{4}$

Neutrino oscillations (1) are the only one amongst these that have been observed in the laboratory. It is sometimes argued that a mass term that would explain can be added "trivially" within the SM. It should, however, be clear that the construction of such a mass term in the framework of renormalisable quantum field theory necessarily requires the introduction of new physical states.

In the SM all fermion masses are Dirac masses, which are generated via the Higgs mechanism from Yukawa couplings, e.g. $\overline{e_{L}} m_{e} e_{R}$ for charged leptons. In order to write down such a term for neutrinos, one necessarily has to add right handed neutrinos $v_{R}$ to the SM Lagrangian $\mathscr{L}_{S M}$. If a Dirac mass $\overline{v_{L}} m_{D} v_{R}$ is the only source of neutrino masses, then the mass generation is exactly the same as for charged leptons and quarks. Then neutrinos are Dirac particles and the lepton sector resembles the quark sector without strong interaction (and with a different choice of the numerical parameters). Then $v_{L}$ and $v_{R}$ form a Dirac spinor $\Psi_{v}=v_{L}+v_{R}$, and the new degrees of freedom $v_{R}$ would not appear as "new particles", but rather lead to additional spin states for the neutrinos. However, as gauge singlets the $v_{R}$ can have a Majorana mass term $\overline{v_{R}} M_{M} v_{R}^{c}$. $M_{M}$ in general is not diagonal in the flavour basis where $m_{D}$ is diagonal. Diagonalising the full mass term leads to Majorana neutrinos and new mass eigenstates. These sterile neutrinos appear as new physical particles that can be responsible for various phenomena, including (2) and (3), see [4] for a recent review. If one wants to avoid this, one has to forbid $M_{M}$ by postulating an additional symmetry (e.g. lepton number conservation).

One could attempt to directly write down a Majorana mass $\overline{v_{L}} m_{v} v_{L}^{c}$ term for the LH neutrinos $v_{L}$, which in this case are Majorana particles. While gauge invariance forbids such a term at

\footnotetext{
${ }^{1}$ Sometimes the acceleration of the cosmic expansion at present time is included in this list as "dark energy". However, all present observational data is consistent with a cosmological constant, which is simply a free parameter in general relativity.

${ }^{2}$ In addition to this empirical evidence, there are a number of aesthetic issues that appear unsatisfactory from a theory viewpoint, such as the hierarchy problem, strong CP-problem, the flavour puzzle and the value of the cosmological constant. Moreover, it is not clear what is the correct description of quantum gravity, Though extremely interesting, this is not required to explain any experiment in foreseeable time.

${ }^{3}$ See [2] for a recent review of the evidence for this interpretation.

${ }^{4}$ The idea of cosmic inflation [3] provides an elegant solution to this problem, but it is not known what mechanism drove the accelerated expansion.
} 
the "fundamental" level, it can be generated via the Higgs mechanism form the Weinberg operator $\frac{1}{2} \overline{L_{L}} \tilde{\phi} f \tilde{\phi}^{T} L_{L}^{c}$ [5] with $m_{v}=v^{2} f M^{-1}$, where $f$ is a flavour matrix, $v$ is the Higgs vev and $M$ characterises mass scales far above the energy of neutrino experiments. Indeed all experimental neutrino data can be explained by adding this operator to the SM. However, the new term is not renormalisable. In a fundamental theory it should be generated by "integrating out" some heavier new states with masses $\sim M$. This could be RH neutrinos $v_{R}$ with a Majorana mass $M_{M}$ (in which case $M \sim M_{M}$ ), see section 3, but there are numerous other possibilities, see e.g. [6] for a summary. Exploring these is the goal of neutrino model building. The physical neutrino mass squares $m_{i}^{2}$ are given by the eigenvalues of $m_{v} m_{v}^{\dagger}$ and can conveniently be read off as $\left|m_{i}\right|$ after diagonalising $m_{v}=U_{v} \operatorname{diag}\left(m_{1}, m_{2}, m_{3}\right) U_{v}^{T}$, where with $U_{v}$ is the neutrino mixing matrix in the charged lepton mass basis. The past few years have seen enormous progress in determining the parameters in $m_{v}$. All three mixing angles and two mass splittings $\left|m_{i}^{2}-m_{j}^{2}\right|$ have been measured, and experimental data may soon allow to constrain the CP-violating Dirac phase [7] and the mass ordering [8].

In contrast, next to nothing is known about the new states that generate $m_{v}$. Though the measurement of the mass splittings and mixings angles is an immense experimental achievement, and the finding of CP-violation in $m_{v}$ would clearly be a milestone achievement in experimental physics, the ultimate goal remains to identify the new states and unveil the mechanism of neutrino mass generation. There are two possible explanations why they have not been found to date. Either they are much heavier than the W-boson ("energy frontier"), or they have very feeble interactions ("intensity frontier"). Of course, a discovery is only possible if the new mass scale $M$ is within reach of experiments, i.e. at the $\mathrm{TeV}$ scale or below, and even then the search might be very challenging. However, a discovery would not only clarify the origin of neutrino masses, but the physics behind their generation may also be responsible for other phenomena including (2) and (3). Hence, neutrino masses may act as a "portal" to a (possibly more complicated) unknown/hidden sector and yield the answer to deep questions in cosmology, such as the origin of matter and dark matter.

\section{Neutrino masses}

Any model of neutrino masses should address the fact that they are orders of magnitude smaller than any other fermion masses in the SM ("mass puzzle"). It is very convenient to classify models according to the way how this hierarchy is explained.

Small coupling constant: A tiny coupling constant can explain the smallness of $m_{i}$ generated via spontaneous symmetry breaking, but it would have to be very tiny. For instance, Dirac masses generated via the standard Higgs mechanism would require $F \sim 10^{-12}$, which is considered "unnatural" by most theorists.

Seesaw mechanism: If the $m_{i}$ are generated at classical level, they may be suppressed by the new heavy scale $M$. The most studied version is the type-I seesaw [9] discussed in section 3, the two other possibilities [10] are the type-II [11] and type-III [12] seesaw.

Flavour ("horizontal") symmetry: Individual entries of the matrix $m_{v}$ may be large, but still yield small $m_{i}$ if there is a symmetry that leads to approximate lepton number conservation and cancellations in $m_{v} m_{v}^{\dagger}$. Prominent examples of this class are Froggatt-Nielsen type models [13], the inverse seesaw [14] and other models with approximate lepton number conservation, e.g. [15]. Radiative masses: Neutrinos could be classically massless and their masses generated by quantum 
corrections. The suppression by the "loop factor" $(4 \pi)^{2}$ is not sufficient to explain the smallness of the $m_{i}$, but the interaction of the new particles in the loop with $v_{L}$ may involve some small coupling constants that do the job. Flavour symmetries or an additional seesaw-like suppression can help to make such models more "natural", see e.g. [16, 17].

Of course, any combination of these ideas could be realised in nature. In addition to the smallness of the mass eigenvalues, it would be desirable to find an explanation for the observed flavour structure of $m_{v}$ ("flavour puzzle"). While the quark mass matrix shows a distinct structure (being approximately diagonal in the weak interaction basis), there is no obvious symmetry in $m_{v}$. Numerous attempts have been made to impose more subtle discrete or continuous symmetries, see e.g. [18]. The basic problem is that the reservoir of possible symmetries is practically unlimited, so for any possible observed $m_{v}$ one could find some kind of symmetry that "predicts" it. Since we already know a lot about the flavour structure, models can only be convincing if they either predict other (yet unmeasured) observables or are "simple" and esthetically convincing from some viewpoint. Prior to the measurement of $\theta_{13}$ there seemed to be at least some kind of structure, and models predicting $\theta_{13}=0$ seemed well-motivated, such as tri/bi-maximal mixing [19]. With the present data, it seems very difficult to single out any class of models that could explain $m_{v}$ in terms of a simple symmetry and/or a small number of parameters, and interest in anarchic models [20] with random values has grown.

In the following I focus on those scenarios in which RH neutrinos $v_{R}$ generate $m_{v}$ via the seesaw mechanism. While the seesaw mechanism alone is unable to predict the flavour structure, it at least explains the smallness of neutrino masses. The existence of $v_{R}$ seems very well-motivated because all other known fermions exist with both, LH and RH chirality. Moreover, $v_{R}$ appear in many popular theories (left-right symmetric models, $\mathrm{SO}(10)$ grand unified theories, generally all theories with a $U(1)_{B-L}$ symmetry) and can be related to various other phenomena in cosmology and particle physics, such as dark matter, baryogenesis, dark radiation and neutrino oscillation anomalies, see $[4,21]$ for an overview.

\section{Probing the Seesaw Mechanism}

The (type-I) seesaw model is defined by adding $n$ neutral fermions $v_{R}$ with RH chirality to the $\mathrm{SM}$. These are referred to as RH neutrinos because they can couple to the SM neutrinos $v_{L}$ in the same way as the RH and LH part of the charged leptons are coupled. The Lagrangian reads

$$
\mathscr{L}=\mathscr{L}_{S M}+i \overline{v_{R}} \partial v_{R}-\overline{l_{L}} F v_{R} \tilde{\Phi}-\tilde{\Phi}^{\dagger} \overline{v_{R}} F^{\dagger} l_{L}-\frac{1}{2}\left(\overline{v_{R}^{c}} M_{M} v_{R}+\overline{v_{R}} M_{M}^{\dagger} v_{R}^{c}\right)
$$

where flavour and isospin indices are suppressed. $\mathscr{L}_{S M}$ is the SM Lagrangian, $l_{L}=\left(v_{L}, e_{L}\right)^{T}$ are the LH lepton doublets and $\Phi$ is the Higgs doublet with $\tilde{\Phi}=\varepsilon \Phi^{*}$, were $\varepsilon$ is the antisymmetric $S U$ (2) tensor. $M_{M}$ is a Majorana mass term for $v_{R}$ with $v_{R}^{c}=C{\overline{v_{R}}}^{T}, 5$ and $F$ is a matrix of Yukawa couplings. We work in a flavour basis with $M_{M}=\operatorname{diag}\left(M_{1}, M_{2}, M_{3}\right)$. For $M_{I}>1 \mathrm{eV}$ there are two distinct sets of mass eigenstates, which we represent by flavour vectors of Majorana spinors $v$ and $N$. The elements $v_{i}$ of the vector

$$
v=V_{v}^{\dagger} v_{L}-U_{v}^{\dagger} \theta v_{R}^{c}+\text { c.c. }
$$

\footnotetext{
${ }^{5}$ The charge conjugation matrix is $C=i \gamma_{2} \gamma_{0}$ in the Weyl basis.
} 
are mostly superpositions of the "active" SU(2) doublet states $v_{L}$ and have light masses $\sim-F^{2} \times$ $v^{2} / M_{I} \ll M_{I}$. Here c.c. stands for the $c$-conjugation defined above. The elements $N_{I}$ of

$$
N=V_{N}^{\dagger} v_{R}+\Theta^{T} v_{L}^{c}+\text { c.c. }
$$

are mostly superpositions of the "sterile" singlet states $v_{R}$ with masses of the order of $M_{I}$. At energies below the electroweak scale, these heavy neutral leptons interact with the SM via their mixing with active neutrinos, which is characterised by the matrix elements $\Theta_{\alpha I} \ll 1$; if kinematically allowed, they participate in all processes in the same way as SM neutrinos, but with cross sections suppressed by $U_{\alpha I}^{2} \equiv\left|\Theta_{\alpha I}\right|^{2} \ll 1 . V_{v}$ is the usual neutrino mixing matrix $V_{v} \equiv\left(\mathbb{1}-\frac{1}{2} \theta \theta^{\dagger}\right) U_{v}$ with $\theta \equiv m_{D} M_{M}^{-1}$, where $m_{D} \equiv F v$. $U_{v}$ is its unitary part, $V_{N}$ and $U_{N}$ are their equivalents in the sterile sector and $\theta \equiv \Theta U_{N}^{T}$. The unitary matrices $U_{v}$ and $U_{N}$ diagonalise the mass matrices

$$
m_{v} \simeq-v^{2} F M_{M}^{-1} F^{T}=-\theta M_{M} \theta^{T}, M_{N} \simeq M_{M}+\frac{1}{2}\left(\theta^{\dagger} \theta M_{M}+M_{M}^{T} \theta^{T} \theta^{*}\right),
$$

respectively. The eigenvalues of $M_{M}$ and $M_{N}$ coincide in very good approximation, and the terms of order $\mathscr{O}\left[\theta^{2}\right]$ are only relevant if two of the $M_{I}$ are quasi-degenerate. If (3.4) is the only source of neutrino masses, then the $n$ must at least equal the number of non-zero $m_{i}$, i.e. $n \geq 2$ if the lightest neutrino is massless and $n \geq 3$ if it is massive. Very little is known about the magnitude of the $M_{I}$. If the $N_{I}$-interactions are to be described by perturbative quantum field theory, then the $M_{I}$ should be at least 1-2 orders of magnitude below the Planck mass. This can be estimated by inserting the observed neutrino mass differences into (3.4). On the lower end they can have eV (or even sub-eV) masses [86]. For $n \geq 3$ any value in between is experimentally allowed [23]. In the following I summarise the most popular seesaw scale choices and their phenomenological implications.

\subsection{The GUT-seesaw}

In the probably most discussed version of the seesaw mechanism the $M_{I}$ are far above the electroweak scale. This choice is primarily theoretically motivated by aesthetic arguments: For "natural" entries (i.e. of order unity) of the $F$, neutrino masses near the upper limit on $\sum_{i} m_{i}<0.23$ imposed by Planck [25] imply values of $M_{I} \sim 10^{14}-10^{15} \mathrm{GeV}$, slightly below the suspected scale of grand unification. Hence, this scenario can easily be embedded in grand unifying theories. Heavy $M_{I}$ are also well-motivated from cosmology. Leptogenesis [26] is one of the most popular explanations for the observed BAU (2). In the most studied version of leptogenesis, the BAU is generated in the CP-violating decay of $N_{I}$, see e.g. [27] for a review. For a non-degenerate mass spectrum and without any degrees of freedom in addition to (3.1), this mechanism only works for $M_{1} \gtrsim 4 \times 10^{8} \mathrm{GeV}$ [29]. Flavour effects [28] can reduce this lower bound by $1-2$ orders of magnitude [30], which is still far out of experimental reach. ${ }^{6}$ If the $M_{I}$ are indeed that large, then the new states $N_{I}$ cannot be found in any near future experiment (and possibly never). The only traces they leave in experiments can be parametrised in terms of higher dimensional operators in an effective Lagrangian obtained after integrating them out [32], including the Weinberg operator with $f=F M_{M}^{-1} F^{T}$. On the positive side, probing these operators allows to constrain some of the parameters in $F$ and $M_{M}$ by looking for rare processes, such as neutrinoless double $\beta$-decay or

\footnotetext{
${ }^{6}$ The consistent description of all quantum and flavour effects remains an active field of research [31].
} 
$\mu \rightarrow e \gamma$, hence indirectly testing physics at a very high scale. For a degenerate $M_{I}$-spectrum or $n=1$ these bounds can indeed be quite strong, see e.g. [33], for $n=3$ they are much weaker [43]. On the negative side, the seesaw mechanism is not the only way to generate these operators, and without directly finding the new states, it is impossible to definitely distinguish it from other scenarios. While leptogenesis may be falsified indirectly [34], the GUT seesaw itself can always escape falsification if the $\mathrm{CP}$-violation is small and the BAU generated from another source. This lack of falsifiability is somewhat unsatisfying, though Nature might not care about this. Moreover, with couplings $F$ of order unity, the $N_{I}$ contribute to the hierarchy problem, and thermal leptogenesis requires a large reheating temperature, which is at tension with upper limits on the temperature in supersymmetric theories [35] ("gravitino problem").

\subsection{The TeV-seesaw}

The highest scale that can be probed directly by collider experiments is the TeV scale. Searches for heavy neutral leptons like $N_{I}$ have been undertaken at the ATLAS and CMS experiments at the Large Hadron Collider (LHC) [36], so far without positive result. These searches have been performed for both, the minimal seesaw (3.1) as well as its left-right symmetric extension, see e.g. [37]. The experimental challenge lies mainly in the fact that the Yukawa interactions $F$ that govern the branching ratios are constrained by the seesaw relation (3.4); a relatively low seesaw scale $M_{I}$ at or below the $\mathrm{TeV}$ scale generally requires very small values of the $F_{\alpha I} \sim F_{0} \equiv\left(m_{i} M_{I} / v^{2}\right)^{1 / 2}$, hence unobservable tiny branching ratios. In the minimal seesaw (3.1) a discovery at the LHC is only realistic if the individual $F_{\alpha I}$ are much bigger than $F_{0}$, and the smallness of the $m_{i}$ is achieved due to a cancellation in the matrix valued equation (3.4) [38-40]. This is realised in models with approximate lepton number conservation, and chances are generally better in extensions of (3.1) in which the $N_{I}$ have additional interactions, see $[14,15]$. If the $M_{I}$ are slightly lower, below the masses of the $\mathrm{W}$ and Z-boson, then $N_{I}$ can be produced in the decay of these gauge bosons. This allows to impose much stronger constraints [42], see [43] for a summary. Possible future direct searches have e.g. been studied in [44]. In addition to direct searches, neutrino oscillation experiments and the bounds on lepton flavour and lepton number violation [45] mentioned in the previous paragraph as well as electroweak precision data [46] impose indirect constraints on the $N_{I}$-properties, see e.g. [41, 43].

Heavy neutrinos $N_{I}$ with $M_{I}$ at the electroweak or TeV scale are also interesting cosmologically because they can generate the BAU via leptogenesis either during their decay [47] or thermal production ("baryogenesis from neutrino oscillations") [48]. For $n=2$ flavours of sterile neutrinos the observed BAU can only be explained in the minimal model (3.1) if it is enhanced by a mass degeneracy [47], for $n=3$ or more flavours no degeneracy is required [49]. This is further relaxed in model $\mathrm{s}$ with additional degrees of freedom [50,51]. Though leading order estimates exist, the quantitative description of these low scale leptogenesis scenarios is highly non-trivial due to the complicated interplay of quantum, thermodynamic and flavour effects and remains an active field of research [52].

\subsection{The GeV-seesaw}

For $M_{I}$ below the mass of the B-mesons the existing constraints and the perspectives for future searches improve significantly (and even more below the D-meson mass), see [41, 43] for a com- 
prehensive overview. On one hand the $N_{I}$ can be produced efficiently in meson decays, allowing to impose upper bounds on the individual $U_{\alpha I}^{2}$, see $[4,24,41,43,53,54]$ and references therein. On the other hand neutrino oscillation data and the seesaw relation (3.4) impose stronger bounds on the sum $U_{I}^{2} \equiv \sum_{\alpha} U_{\alpha I}^{2}$. Finally, the requirement to decay before BBN [23] imposes a strict lower bound on $U_{I}^{2}$ as a function of $M_{I}[43,55]$. There are constraints from lepton flavour violation $[43,56]$ neutrinoless double $\beta$-decay $[43,45,57]$ and lepton universality in meson decays $[43,58]$. For $N_{I}$ heavier than D-mesons, they can be more constraining than direct search bounds [43].

For $M_{I}$ in the $\mathrm{GeV}$ range, the BAU can be explained via leptogenesis during the thermal production of the $N_{I}[48,59,60,62]$. Similarly to the TeV scale, this requires a mass degeneracy nor $n=2$ [61, 62], but no such degeneracy is needed for $n=3$ [63]. The leptogenesis parameter space will be further explored in the near future. For $M_{I}$ below the D-meson mass this is e.g. done by the NA62 experiment, for heavier masses LHCb and BELLE II will improve the bounds [54]. The ideal tool to search for $N_{I}$ in the $\mathrm{GeV}$ range would be the proposed SHiP experiment [64]. In a small fraction of the parameter space the CP-violation responsible for the BAU comes from the "Dirac phase" in $U_{v}$ that may be measured in neutrino oscillation experiments [62], but in general it lies in the sterile sector and can only be measured in $N_{I}$ decays if their mass spectrum is degenerate [65].

\subsection{The keV-seesaw}

Sterile neutrinos $N_{I}$ are massive, feebly interacting and can be very long lived. This makes them obvious DM candidates $[66,75,76]$. Their properties are constrained by astrophysical, cosmological and laboratory data. Most importantly, the radiative decay $N \rightarrow v \gamma$ would lead to an observable photon emission line at energy $M_{I} / 2$ from DM dense regions [77]. Until 2014, the nonobservation of an emission line could only be use to impose an upper bound on $U_{I}^{2}$ as a function of $M_{I}$. These "established constraints" are e.g. discussed in $[4,67]$ and references therein; they imply that the $N_{I}$ that compose the DM must be so feebly coupled that they cannot contribute significantly to the neutrino masses (3.4) or leptogenesis. In 2014, two groups reported an unexplained emission signal at $\sim 3.5 \mathrm{keV}[68]$ that can be interpreted as evidence for sterile neutrino DM, though this interpretation is disputed [69]. Observations with the Astro-H satellite [70] may help to clarify the situation. Since thermal production via mixing is unavoidable [66], an upper bound on $U_{I}^{2}$ can also be obtained from the requirement not to produce too much DM. The DM mass $M_{I}$ is bound from below by phase space considerations (essentially Pauli's exclusion principle in DM dense regions) [71]. In the laboratory, $U_{I}^{2}$ can be constrained by KATRIN and similar experiments [72]. All other constraints depend on the mechanism by which the $N_{I}$ are produced in the early universe. Thermal production via mixing is most efficient at temperatures $T \sim 100 \mathrm{MeV}$ [73] and always leads to a $N_{I}$ population with a thermal momentum distribution, but a total abundance far below the equilibrium value. X-ray bounds force $M_{I}$ to be in the $\mathrm{keV}$ range, which can be realised in different models [74]. This "warm DM" component cannot compose all the DM, as their mean free path during structure formation in the early universe would be in tension with the observed small scale structure in the universe, see e.g. [4, 67] and references therein. However, in the presence of a significant lepton asymmetry, there is also a resonantly produced "cold" component [75] produced due to the MSW effect, which allows to explain all DM in terms of sterile neutrinos in agreement with structure formation bounds [78]. Interestingly, the three phenomena (1)-(3) can be explained 
simultaneously within the minimal model (3.1) if one of the $N_{I}$ has a $\mathrm{keV}$ mass and acts as DM while the other two have degenerate masses in the $\mathrm{GeV}$ range and are responsible for leptogenesis and neutrino masses [24]. This scenario, first proposed in [59], was shown to be feasible in [62]. A cold component can also be produced nonthermally, e.g. due to a coupling to an inflaton [79], the SM Higgs [81], other scalars [82] or modified gravity [83]. Then the mass can be larger than keV because the production for not rely on the mixing $\theta$, which can in turn be made arbitrarily small to ensure a long lifetime even for a large mass. Finally, the spectrum can effectively be "cooled down" if entropy is injected into the rest of the primordial plasma due the decay of some heavy particle, which allows to reconcile an initially warm or hot DM spectrum with structure formation constraints [84]. Finally, it has been proposed that keV mass sterile neutrinos are responsible for pulsar kicks [85].

\subsection{The (sub)eV-seesaw}

In principle neutrino oscillation data can be explained via the seesaw mechanism with $M_{I}$ as low as an eV [86]. For even lower masses the seesaw hierarchies $M_{I} \gg m_{i}$ and $\theta \ll 1$ do not hold, and for $M_{I} \ll m_{i}$ one effectively has Dirac neutrinos. This case requires $M_{I}<10^{-9} \mathrm{eV}$, otherwise solar neutrino oscillations into $v_{R}$ should have been observed [87]. Sterile neutrinos with $M_{I}$ in the eV range could also explain the LSND [88], Gallium [89] and reactor anomalies [90] and/or act as extra relativistic degrees of freedom in the early universe ("dark radiation"). Note, however, that light sterile neutrinos that explain these anomalies cannot simultaneously explain the masses of active neutrinos; they would have to be added on top of the usual seesaw (3.4) While all these anomalies as well as some cosmological data sets seem to favour the existence of light sterile neutrinos, there is no convincing model that can fit all data sets simultaneously without significant tension. On the other hand, the statistical significance is not sufficient to rule out their existence, even if the recent Planck data (which disfavours light sterile neutrinos [25]) is taken into account. More detailed discussions can e.g. be found in [4, 21], see [91, 92] for recent updates. Ultimately this question can only be clarified by new experiments.

\section{Conclusion}

To date, neutrino oscillations are the only established evidence for the existence of new physical states that has been found in the laboratory. The recent years have seen immense progress in the determination of the neutrino mixing angles and mass splittings. There are several important remaining questions about the properties of neutrinos that (with some luck) can be answered in foreseeable time, including the absolute mass scale, the nature of their mass term (Dirac vs Majorana) and the presence of CP-violation in the lepton sector. The ultimate goal, however, remains to unveil the mechanism of neutrino mass generation and to identify the new physical states that are involved in it, which could provide the key to understand other unsolved puzzles in both, cosmology and particle physics. Unfortunately there is no guarantee that this can be achieved in foreseeable time. In spite of this, it is important to explore the neutrino sector in as much detail as possible. Given the present lack of any new physics signals from high energy experiments, it remains the only probe of new physics that can be studied in the laboratory. 


\section{References}

[1] K. A. Olive et al. [Particle Data Group Collaboration], Chin. Phys. C 38 (2014) 090001.

[2] L. Canetti, M. Drewes and M. Shaposhnikov, New J. Phys. 14 (2012) 095012 [arXiv:1204.4186 [hep-ph]].

[3] A. A. Starobinsky, Phys. Lett. B 91 (1980) 99; A. H. Guth, Phys. Rev. D 23 (1981) 347; A. D. Linde, Phys. Lett. B 108 (1982) 389.

[4] M. Drewes, Int. J. Mod. Phys. E 22 (2013) 1330019 [arXiv:1303.6912 [hep-ph]].

[5] S. Weinberg, Phys. Rev. Lett. 43 (1979) 1566.

[6] R. N. Mohapatra and P. B. Pal, World Sci. Lect. Notes Phys. 60 (1998) 1 [World Sci. Lect. Notes Phys. 72 (2004) 1]; M. Fukugita and T. Yanagida, Berlin, Germany: Springer (2003) 593 p.; E. Ma, arXiv:0905.0221 [hep-ph]; A. Strumia and F. Vissani, hepph/0606054; R. N. Mohapatra, S. Antusch, K. S. Babu, G. Barenboim, M. C. Chen, A. de Gouvea, P. de Holanda and B. Dutta et al., Rept. Prog. Phys. 70 (2007) 1757 [hep-ph/0510213].

[7] M. C. Gonzalez-Garcia, M. Maltoni and T. Schwetz, JHEP 1411 (2014) 052 [arXiv:1409.5439 [hep-ph]]; F. Capozzi, G. L. Fogli, E. Lisi, A. Marrone, D. Montanino and A. Palazzo, Phys. Rev. D 89 (2014) 093018 [arXiv:1312.2878 [hep-ph]].

[8] M. Blennow, P. Coloma, P. Huber and T. Schwetz, JHEP 1403 (2014) 028 [arXiv:1311.1822 [hep-ph]].

[9] P. Minkowski, Phys. Lett. B 67 (1977) 421: M. Gell-Mann, P. Ramond and R. Slansky, Conf. Proc. C 790927 (1979) 315 [arXiv:1306.4669 [hep-th]]; R. N. Mohapatra and G. Senjanovic, Phys. Rev. Lett. 44 (1980) 912; T. Yanagida, Prog. Theor. Phys. 64 (1980) 1103.

[10] E. Ma, Phys. Rev. Lett. 81 (1998) 1171 [hep-ph/9805219].

[11] T. P. Cheng and L. F. Li, Phys. Rev. D 22 (1980) 2860; J. Schechter and J. W. F. Valle, Phys. Rev. D 22 (1980) 2227; G. Lazarides, Q. Shafi and C. Wetterich, Nucl. Phys. B 181 (1981) 287; R. N. Mohapatra and G. Senjanovic, Phys. Rev. D 23 (1981) 165. M. Magg and C. Wetterich, Phys. Lett. B 94 (1980) 61.

[12] R. Foot, H. Lew, X. G. He and G. C. Joshi, Z. Phys. C 44 (1989) 441.

[13] C. D. Froggatt and H. B. Nielsen, Phys. Lett. B 368 (1996) 96 [hep-ph/9511371].

[14] R. N. Mohapatra and J. W. F. Valle, Phys. Rev. D 34 (1986) 1642; R. N. Mohapatra, Phys. Rev. Lett. 56 (1986) 561.

[15] Y. Chikashige, R. N. Mohapatra and R. D. Peccei, Phys. Lett. B 98 (1981) 265; G. B. Gelmini and M. Roncadelli, Phys. Lett. B 99 (1981) 411; D. Wyler and L. Wolfenstein, Nucl. Phys. B 218 (1983) 205; M. C. Gonzalez-Garcia and J. W. F. Valle, Phys. Lett. B 216 (1989) 360; G. C. Branco, W. Grimus and L. Lavoura, Phys. Lett. B 380 (1996) 119 [hep-ph/9601383]; A. Abada, C. Biggio, F. Bonnet, M. B. Gavela and T. Hambye, JHEP 0712 (2007) 061 [arXiv:0707.4058 [hep-ph]]; M. Shaposhnikov, Nucl. Phys. B 763 (2007) 49 [hep-ph/0605047]; M. B. Gavela, T. Hambye, D. Hernandez and P. Hernandez, JHEP 0909 (2009) 038 [arXiv:0906.1461 [hep-ph]].

[16] A. Zee, Phys. Lett. B 93 (1980) 389 [Erratum-ibid. B 95 (1980) 461]; E. Witten, Phys. Lett. B 91 (1980) 81. A. Zee, Nucl. Phys. B 264 (1986) 99; K. S. Babu, Phys. Lett. B 203 (1988) 132; E. Ma, Phys. Rev. D 73 (2006) 077301 [hep-ph/0601225];

[17] V. V. Khoze and G. Ro, JHEP 1310 (2013) 075 [arXiv:1307.3764].

[18] S. F. King, A. Merle, S. Morisi, Y. Shimizu and M. Tanimoto, New J. Phys. 16 (2014) 045018 [arXiv:1402.4271 [hep-ph]].

[19] P. F. Harrison, D. H. Perkins and W. G. Scott, Phys. Lett. B 458 (1999) 79 [hep-ph/9904297]; P. F. Harrison and W. G. Scott, Phys. Lett. B 535 (2002) 163 [hep-ph/0203209]; P. F. Harrison, D. H. Perkins and W. G. Scott, Phys. Lett. B 530 (2002) 167 [hep-ph/0202074]; E. Ma, Phys. Rev. D 70 (2004) 031901 [hep-ph/0404199]; G. Altarelli and F. Feruglio, Nucl. Phys. B 741 (2006) 215 [hep-ph/0512103]; G. Altarelli and F. Feruglio, Nucl. Phys. B 720 (2005) 64 [hep-ph/0504165]; Z. z. Xing, Phys. Lett. B 533 (2002) 85 [hep-ph/0204049].

[20] L. J. Hall, H. Murayama and N. Weiner, Phys. Rev. Lett. 84 (2000) 2572 [hep-ph/9911341].

[21] K. N. Abazajian, M. A. Acero, S. K. Agarwalla, A. A. Aguilar-Arevalo, C. H. Albright, S. Antusch, C. A. Arguelles and A. B. Balantekin et al., arXiv:1204.5379 [hep-ph].

[22] A. de Gouvea, Phys. Rev. D 72 (2005) 033005 [hep-ph/0501039].

[23] P. Hernandez, M. Kekic and J. Lopez-Pavon, Phys. Rev. D 90 (2014) 6, 065033 [arXiv:1406.2961 [hep-ph]].

[24] A. Boyarsky, O. Ruchayskiy and M. Shaposhnikov, Ann. Rev. Nucl. Part. Sci. 59 (2009) 191 [arXiv:0901.0011 [hep-ph]].

[25] P. A. R. Ade et al. [Planck Collaboration], Astron. Astrophys. 571 (2014) A16 [arXiv:1303.5076 [astro-ph.CO]].

[26] M. Fukugita and T. Yanagida, Phys. Lett. B 174 (1986) 45.

[27] W. Buchmuller, R. D. Peccei and T. Yanagida, Ann. Rev. Nucl. Part. Sci. 55 (2005) 311 [hep-ph/0502169]; S. Davidson, E. Nardi and Y. Nir, Phys. Rept. 466 (2008) 105 [arXiv:0802.2962 [hep-ph]].

[28] R. Barbieri, P. Creminelli, A. Strumia and N. Tetradis, Nucl. Phys. B 575 (2000) 61 [hep-ph/9911315]; A. Abada, S. Davidson, A. Ibarra, F.-X. Josse-Michaux, M. Losada and A. Riotto, JHEP 0609 (2006) 010 [hep-ph/0605281]; A. Abada, S. Davidson, F. X. Josse-Michaux, M. Losada and A. Riotto, JCAP 0604 (2006) 004 [hep-ph/0601083]; E. Nardi, Y. Nir, E. Roulet and J. Racker, JHEP 0601 (2006) 164 [hep-ph/0601084]; S. Blanchet and P. Di Bari, JCAP 0703 (2007) 018 [hep-ph/0607330].

[29] S. Davidson and A. Ibarra, Phys. Lett. B 535 (2002) 25 [hep-ph/0202239].

[30] S. Antusch, S. Blanchet, M. Blennow and E. Fernandez-Martinez, JHEP 1001 (2010) 017 [arXiv:0910.5957 [hep-ph]]; J. Racker, M. Pena and N. Rius, JCAP 1207 (2012) 030 [arXiv:1205.1948 [hep-ph]].

[31] V. Cirigliano, C. Lee, M. J. Ramsey-Musolf and S. Tulin, Phys. Rev. D 81 (2010) 103503 [arXiv:0912.3523 [hep-ph]].; C. Fidler, M. Herranen, K. Kainulainen and P. M. Rahkila, JHEP 1202 (2012) 065 [arXiv:1108.2309 [hep-ph]]; M. Beneke, B. Garbrecht, C. Fidler, M. Herranen and P. Schwaller, Nucl. Phys. B 843 (2011) 177 [arXiv:1007.4783 [hep-ph]]; M. Beneke, B. Garbrecht, M. Herranen and P. Schwaller, Nucl. Phys. B 838 (2010) 1 [arXiv:1002.1326 [hep-ph]]; A. Anisimov, W. Buchmuller, 
M. Drewes and S. Mendizabal, Phys. Rev. Lett. 104 (2010) 121102 [arXiv:1001.3856 [hep-ph]]; A. Anisimov, W. Buchm§ller, M. Drewes and S. Mendizabal, Annals Phys. 326 (2011) 1998 [Erratum-ibid. 338 (2011) 376] [arXiv:1012.5821 [hep-ph]]; T. Frossard, M. Garny, A. Hohenegger, A. Kartavtsev and D. Mitrouskas, Phys. Rev. D 87 (2013) 085009 [arXiv:1211.2140 [hep-ph]].

[32] A. Broncano, M. B. Gavela and E. E. Jenkins, Phys. Lett. B 552 (2003) 177 [Erratum-ibid. B 636 (2006) 330] [hep-ph/0210271].

[33] M. Blennow, E. Fernandez-Martinez, J. Lopez-Pavon and J. Menendez, JHEP 1007 (2010) 096 [arXiv:1005.3240 [hep-ph]].

[34] F. F. Deppisch, J. Harz and M. Hirsch, Phys. Rev. Lett. 112 (2014) 221601 [arXiv:1312.4447 [hep-ph]].

[35] H. Pagels and J. R. Primack, Phys. Rev. Lett. 48 (1982) 223.

[36] G. Aad et al. [ATLAS Collaboration], Eur. Phys. J. C 72 (2012) 2056 [arXiv:1203.5420 [hep-ex]]; V. Khachatryan et al. [CMS Collaboration], Eur. Phys. J. C 74 (2014) 11, 3149 [arXiv:1407.3683 [hep-ex]].

[37] J. Barry and W. Rodejohann, JHEP 1309 (2013) 153 [arXiv:1303.6324 [hep-ph]]; C. Y. Chen, P. S. B. Dev and R. N. Mohapatra, Phys. Rev. D 88 (2013) 033014 [arXiv:1306.2342 [hep-ph]].

[38] A. Ibarra, E. Molinaro and S. T. Petcov, Phys. Rev. D 84 (2011) 013005 [arXiv:1103.6217 [hep-ph]].

[39] J. Kersten and A. Y. Smirnov, Phys. Rev. D 76 (2007) 073005 [arXiv:0705.3221 [hep-ph]]; G. Bambhaniya, S. Khan, P. Konar and T. Mondal, arXiv:1411.6866 [hep-ph].

[40] A. Das and N. Okada, Phys. Rev. D 88 (2013) 11, 113001 [arXiv:1207.3734 [hep-ph]].

[41] A. Atre, T. Han, S. Pascoli and B. Zhang, JHEP 0905 (2009) 030 [arXiv:0901.3589 [hep-ph]].

[42] P. Abreu et al. [DELPHI Collaboration], Z. Phys. C 74 (1997) 57 [Erratum-ibid. C 75 (1997) 580]; O. Adriani et al. [L3 Collaboration], Phys. Lett. B 295 (1992) 371.

[43] M. Drewes and B. Garbrecht, arXiv:1502.00477 [hep-ph].

[44] A. Blondel et al. [team for the FCC-ee study Collaboration], arXiv:1411.5230 [hep-ex]; A. Abada, V. De Romeri, S. Monteil, J. Orloff and A. M. Teixeira, arXiv:1412.6322 [hep-ph].

[45] J. Lopez-Pavon, S. Pascoli and C. f. Wong, Phys. Rev. D 87 (2013) 9, 093007 [arXiv:1209.5342 [hep-ph]]; T. Asaka and S. Eijima, PTEP 2013 (2013) 11, 113B02 [arXiv:1308.3550 [hep-ph]].

[46] F. del Aguila, J. de Blas and M. Perez-Victoria, Phys. Rev. D 78 (2008) 013010 [arXiv:0803.4008 [hep-ph]]; E. Akhmedov, A. Kartavtsev, M. Lindner, L. Michaels and J. Smirnov, JHEP 1305 (2013) 081 [arXiv:1302.1872 [hep-ph]].

[47] A. Pilaftsis and T. E. J. Underwood, Nucl. Phys. B 692 (2004) 303 [hep-ph/0309342].

[48] E. K. Akhmedov, V. A. Rubakov and A. Y. Smirnov, Phys. Rev. Lett. 81 (1998) 1359 [hep-ph/9803255].

[49] B. Garbrecht, Phys. Rev. D 90 (2014) 063522 [arXiv:1401.3278 [hep-ph]].

[50] S. K. Kang and A. Patra, arXiv:1412.4899 [hep-ph]; J. Racker, arXiv:1410.5482 [hep-ph];

[51] F. Bezrukov, A. Kartavtsev and M. Lindner, J. Phys. G 40 (2013) 095202 [arXiv:1204.5477 [hep-ph]]; M. Frigerio and C. E. Yaguna, arXiv: 1409.0659 [hep-ph].

[52] M. Garny, A. Kartavtsev and A. Hohenegger, Annals Phys. 328 (2013) 26 [arXiv:1112.6428 [hep-ph]]; B. Garbrecht and M. Herranen, Nucl. Phys. B 861 (2012) 17 [arXiv:1112.5954 [hep-ph]]; S. Iso, K. Shimada and M. Yamanaka, JHEP 1404 (2014) 062 [arXiv:1312.7680 [hep-ph]]; P. S. Bhupal Dev, P. Millington, A. Pilaftsis and D. Teresi, Nucl. Phys. B 891 (2015) 128 [arXiv:1410.6434 [hep-ph]]; B. Garbrecht, F. Gautier and J. Klaric, JCAP 1409 (2014) 09, 033 [arXiv:1406.4190 [hep-ph]].

[53] D. Gorbunov and M. Shaposhnikov, JHEP 0710 (2007) 015 [Erratum-ibid. 1311 (2013) 101] [arXiv:0705.1729 [hep-ph]].

[54] L. Canetti, M. Drewes and B. Garbrecht, Phys. Rev. D 90 (2014) 12, 125005 [arXiv:1404.7114 [hep-ph]].

[55] A. D. Dolgov, S. H. Hansen, G. Raffelt and D. V. Semikoz, Nucl. Phys. B 580 (2000) 331 [hep-ph/0002223]; O. Ruchayskiy and A. Ivashko, JCAP 1210 (2012) 014 [arXiv:1202.2841 [hep-ph]].

[56] L. Canetti and M. Shaposhnikov, Hyperfine Interact. 214 (2013) 1-3, 5; D. Gorbunov and I. Timiryasov, arXiv:1412.7751 [hep-ph].

[57] F. L. Bezrukov, Phys. Rev. D 72 (2005) 071303 [hep-ph/0505247]; T. Asaka and S. Eijima, PTEP 2013 (2013) 11, 113B02 [arXiv:1308.3550 [hep-ph]].

[58] A. Abada, D. Das, A. M. Teixeira, A. Vicente and C. Weiland, JHEP 1302 (2013) 048 [arXiv:1211.3052 [hep-ph]]; A. Abada, A. M. Teixeira, A. Vicente and C. Weiland, JHEP 1402 (2014) 091 [arXiv:1311.2830 [hep-ph]]; L. Basso, O. Fischer and J. J. van der Bij, Europhys. Lett. 105 (2014) 1, 11001 [arXiv:1310.2057 [hep-ph]]; M. Endo and T. Yoshinaga, arXiv:1404.4498 [hep-ph]; T. Asaka, S. Eijima and K. Takeda, arXiv:1410.0432 [hep-ph].

[59] T. Asaka and M. Shaposhnikov, Phys. Lett. B 620 (2005) 17 [hep-ph/0505013]; T. Asaka, S. Blanchet and M. Shaposhnikov, Phys. Lett. B 631 (2005) 151 [hep-ph/0503065].

[60] L. Canetti and M. Shaposhnikov, JCAP 1009 (2010) 001 [arXiv:1006.0133 [hep-ph]];

[61] B. Shuve and I. Yavin, Phys. Rev. D 89 (2014) 075014 [arXiv:1401.2459 [hep-ph]].

[62] L. Canetti, M. Drewes and M. Shaposhnikov, Phys. Rev. Lett. 110 (2013) 6, 061801 [arXiv:1204.3902 [hep-ph]]; L. Canetti, M. Drewes, T. Frossard and M. Shaposhnikov, Phys. Rev. D 87 (2013) 9, 093006 [arXiv:1208.4607 [hep-ph]].

[63] M. Drewes and B. Garbrecht, JHEP 1303 (2013) 096 [arXiv:1206.5537 [hep-ph]].

[64] W. Bonivento, A. Boyarsky, H. Dijkstra, U. Egede, M. Ferro-Luzzi, B. Goddard, A. Golutvin and D. Gorbunov et al., arXiv:1310.1762 [hep-ex].

[65] G. Cvetic, C. S. Kim and J. Zamora-SaĞ, Phys. Rev. D 89 (2014) 093012 [arXiv:1403.2555 [hep-ph]].

[66] S. Dodelson and L. M. Widrow, Phys. Rev. Lett. 72 (1994) 17 [hep-ph/9303287].

[67] A. Boyarsky, D. Iakubovskyi and O. Ruchayskiy, Phys. Dark Univ. 1 (2012) 136 [arXiv:1306.4954 [astro-ph.CO]]. 
[68] E. Bulbul, M. Markevitch, A. Foster, R. K. Smith, M. Loewenstein and S. W. Randall, Astrophys. J. 789 (2014) 13 [arXiv:1402.2301 [astro-ph.CO]]; A. Boyarsky, O. Ruchayskiy, D. Iakubovskyi and J. Franse, Phys. Rev. Lett. 113 (2014) 251301 [arXiv:1402.4119 [astro-ph.CO]].

[69] T. E. Jeltema and S. Profumo, arXiv:1408.1699 [astro-ph.HE]; S. Riemer-Sorensen, arXiv:1405.7943 [astro-ph.CO]; M. E. Anderson, E. Churazov and J. N. Bregman, arXiv:1408.4115 [astro-ph.HE]; A. Boyarsky, J. Franse, D. Iakubovskyi and O. Ruchayskiy, arXiv:1408.4388 [astro-ph.CO]; E. Bulbul, M. Markevitch, A. R. Foster, R. K. Smith, M. Loewenstein and S. W. Randall, arXiv:1409.4143 [astro-ph.HE]; J. M. Cline and A. R. Frey, Phys. Rev. D 90 (2014) 123537 [arXiv:1410.7766 [astro-ph.CO]]; O. Urban, N. Werner, S. W. Allen, A. Simionescu, J. S. Kaastra and L. E. Strigari, arXiv:1411.0050 [astroph.CO]; E. Carlson, T. Jeltema and S. Profumo, arXiv:1411.1758 [astro-ph.HE]; T. Jeltema and S. Profumo, arXiv:1411.1759 [astro-ph.HE].

[70] T. Takahashi, K. Mitsuda, R. Kelley, H. Aharonian, F. Aarts, H. Akamatsu, F. Akimoto and S. Allen et al., Proc. SPIE Int. Soc. Opt. Eng. 8443 (2012) 1 Z [arXiv:1210.4378 [astro-ph.IM]].

[71] D. Gorbunov, A. Khmelnitsky and V. Rubakov, JCAP 0810 (2008) 041 [arXiv:0808.3910 [hep-ph]].

[72] S. Mertens, T. Lasserre, S. Groh, G. Drexlin, F. Glueck, A. Huber, A. W. P. Poon and M. Steidl et al., arXiv:1409.0920 [physics.ins-det]; J. Barry, J. Heeck and W. Rodejohann, JHEP 1407 (2014) 081 [arXiv:1404.5955 [hep-ph]].

[73] T. Asaka, M. Laine and M. Shaposhnikov, JHEP 0701 (2007) 091 [hep-ph/0612182]; T. Asaka, M. Laine and M. Shaposhnikov, JHEP 0606 (2006) 053 [hep-ph/0605209].

[74] A. Merle, Int. J. Mod. Phys. D 22 (2013) 1330020 [arXiv:1302.2625 [hep-ph]].

[75] X. D. Shi and G. M. Fuller, Phys. Rev. Lett. 82 (1999) 2832 [astro-ph/9810076]; K. Abazajian and S. M. Koushiappas, Phys. Rev. D 74 (2006) 023527 [astro-ph/0605271]; M. Laine and M. Shaposhnikov, JCAP 0806 (2008) 031 [arXiv:0804.4543 [hep-ph]].

[76] K. Abazajian, G. M. Fuller and M. Patel, Phys. Rev. D 64 (2001) 023501 [astro-ph/0101524].

[77] K. Abazajian, G. M. Fuller and W. H. Tucker, Astrophys. J. 562 (2001) 593 [astro-ph/0106002].

[78] A. Boyarsky, J. Lesgourgues, O. Ruchayskiy and M. Viel, JCAP 0905 (2009) 012 [arXiv:0812.0010 [astro-ph]].

[79] M. Shaposhnikov and I. Tkachev, Phys. Lett. B 639 (2006) 414 [hep-ph/0604236]; A. Anisimov, Y. Bartocci and F. L. Bezrukov, Phys. Lett. B 671 (2009) 211 [arXiv:0809.1097 [hep-ph]];

[80] F. Bezrukov and D. Gorbunov, JHEP 1005 (2010) 010 [arXiv:0912.0390 [hep-ph]].

[81] F. Bezrukov, D. Gorbunov and M. Shaposhnikov, JCAP 0906 (2009) 029 [arXiv:0812.3622 [hep-ph]].

[82] K. Petraki and A. Kusenko, Phys. Rev. D 77 (2008) 065014 [arXiv:0711.4646 [hep-ph]]; I. M. Shoemaker, K. Petraki and A. Kusenko, JHEP 1009 (2010) 060 [arXiv:1006.5458 [hep-ph]]; F. Bezrukov, A. Kartavtsev and M. Lindner, J. Phys. G 40 (2013) 095202 [arXiv:1204.5477 [hep-ph]].

[83] D. S. Gorbunov and A. G. Panin, Phys. Lett. B 700 (2011) 157 [arXiv:1009.2448 [hep-ph]].

[84] F. Bezrukov, H. Hettmansperger and M. Lindner, Phys. Rev. D 81 (2010) 085032 [arXiv:0912.4415 [hep-ph]]; M. Nemevsek, G. Senjanovic and Y. Zhang, JCAP 1207 (2012) 006 [arXiv:1205.0844 [hep-ph]]; T. Tsuyuki, Phys. Rev. D 90 (2014) 013007 [arXiv:1403.5053 [hep-ph]].

[85] A. Kusenko and G. Segre, Phys. Rev. D 59 (1999) 061302 [astro-ph/9811144]; G. M. Fuller, A. Kusenko, I. Mocioiu and S. Pascoli, Phys. Rev. D 68 (2003) 103002 [astro-ph/0307267].

[86] A. de Gouvea, Phys. Rev. D 72 (2005) 033005 [hep-ph/0501039].

[87] A. de Gouvea, W. C. Huang and J. Jenkins, Phys. Rev. D 80 (2009) 073007 [arXiv:0906.1611 [hep-ph]].

[88] C. Athanassopoulos et al. [LSND Collaboration], Phys. Rev. Lett. 77 (1996) 3082 [nucl-ex/9605003]; C. Athanassopoulos et al. [LSND Collaboration], Phys. Rev. Lett. 81 (1998) 1774 [nucl-ex/9709006].

[89] J. N. Abdurashitov, V. N. Gavrin, S. V. Girin, V. V. Gorbachev, P. P. Gurkina, T. V. Ibragimova, A. V. Kalikhov and N. G. Khairnasov et al., Phys. Rev. C 73, 045805 (2006) [nucl-ex/0512041].

[90] G. Mention, M. Fechner, T. Lasserre, T. A. Mueller, D. Lhuillier, M. Cribier and A. Letourneau, Phys. Rev. D 83 (2011) 073006 [arXiv:1101.2755 [hep-ex]].

[91] B. Leistedt, H. V. Peiris and L. Verde, Phys. Rev. Lett. 113 (2014) 041301 [arXiv:1404.5950 [astro-ph.CO]]; J. Hamann and J. Hasenkamp, JCAP 1310 (2013) 044 [arXiv:1308.3255 [astro-ph.CO]].

[92] J. Kopp, P. A. N. Machado, M. Maltoni and T. Schwetz, PoS Neutel 2013 (2013) 019. 